\title{
ARMY \\ ADVANCED COMMUNICATIONS TECHNOLOGY SATELLITE (ACTS) MOBILE TERMINAL (AAMT)
}

\author{
Thomas C. Jedrey, Major Robert T. Reilander, and Aubrey L. Leyda
}

\begin{abstract}
Thomas C. Jedrey, Jet Propulsion Laboratory, California Institute of Technology, Pasadena, California Major Robert T. Reilander, U.S. Army Communications Electronics Command, Fort Monmouth, New Jersey Aubrey L. Leyda, U.S. Army Signal Center, Fort Gordon, Georgia
\end{abstract}

\begin{abstract}
The NASA Advanced Communications Technology Satellite (ACTS) operates at $20 / 30 \mathrm{GHz}$. It is scheduled for launch in the summer of 1993. The Army will experiment with ACTS to demonstrate new concepts and capabilities in support of Army AirLand Operations Doctrine. Although operating at commercial frequencies, there is much to be learned from ACTS in terms of quantifying and qualifying millimeter wavelength requirements. The role of next generation commercial satellites, and their relationship to the Military Satellite Communications (MILSATCOM) backbone, also bears investigation.
\end{abstract}

One particular area of interest with ACTS is its ability to support communications on the move. The National Aeronautics and Space Administration (NASA), through the Jet Propulsion Laboratory (JPL), has already developed a commercially oriented ACTS Mobile Terminal (AMT). This vehicular terminal employs a mechanically-steered antenna, and can support either digital links up to $64 \mathrm{kbps}$ or analog links. The Army has now contracted JPL to modify this terminal for Army demonstration purposes. The AMT hardware will be mounted on a High Mobility Multipurpose Wheeled Vehicle (HMMWV). In addition to providing standard baseline AMT capabilities, a coupling to a Single Channel Ground Air Radio System (SINCGARS) radio will enable secure command and control on the move. The rationale, design, features, and planned demonstration program for this Army AMT (AAMT) are discussed in this paper.

\section{INTRODUCTION}

As demonstrated by the recent Persian Gulf conflict, the communications requirements of a highly mobile Army can rapidly exceed the capabilities of existing terrestrial and spacebased systems. In addition, the Army's new AirLand Operations Doctrine places a great dependence on improved mobility in order to cope with non-linear and highly dispersed battlefields. The supporting communications infrastructure must be equally responsive. Declining budgets in the face of increased global responsibility make it increasingly difficult for MILSATCOM requirements to be addressed exclusively by Department of Defense (DoD) systems. It is both now necessary and desirable to leverage complementary commercial systems from other government agencies and industry. Such systems must be examined and utilized as much as possible. NASA's ACTS [1] and AMT [2] programs are classic examples of such programs. They represent an opportunity to examine the state-of-the-art in commercial mobile communications. The operational and doctrinal lessons learned from a demonstration of these technologies would be helpful in the definition and clarification of Army mobile communications requirements. The technological experience of such an investigation could well impact the structure of future MILSATCOM systems, or may evolve into follow-on commercial systems that better meet Army Doctrinal needs.

The specific requirements for Army mobile communications systems have traditionally been difficult to quantify and qualify. As a minimum, terminals should be mobile, small, low cost, and capable of providing secure voice and data. Such terminals should also be distance insensitive, meaning that a spacebased solution is likely. JPL's AMT meets all of 
these basic requirements.

The AMT experimentation and demonstration system addresses Army requirements by leveraging NASA developed technologies in satellites, terminals, and subsystems to evaluate and demonstrate $\mathrm{K} / \mathrm{Ka}$-band mobile satellite communications. The AMT is a technology testbed configured to work exclusively with ACTS. The system consists of a fixed terminal communicating with a mobile terminal through ACTS, as illustrated in Figure 1. The user interface to both terminals will be either the AMT speech codec or the SINCGARS [3] radio - currently in the Army's inventory. All standard functions of the SINCGARS radio are expected to be supported, allowing system demonstrations employing military procedures and protocols. To the greatest extent possible, the satellitebased nature of the communications system will be transparent to the end user.

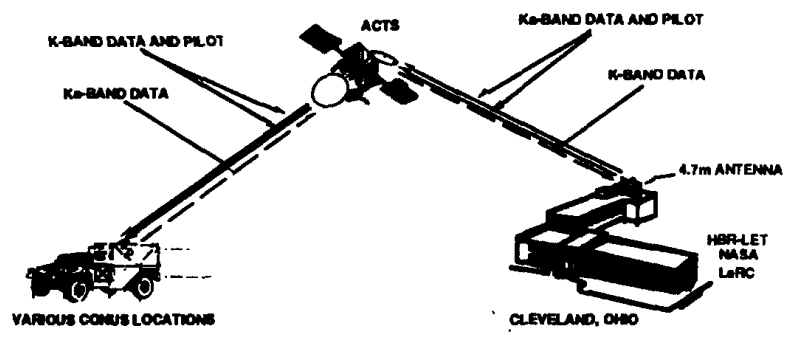

Figure 1 Experimental System Configuration

\section{ARMY ACTS MOBILE TERMINAL (AAMT) SYSTEM DESCRIPTION}

The AAMT system description includes the ACTS, the AMT, modifications to the AMT for the AAMT, and a fixed base station terminal. As illustrated in Figure 1, the fixed terminal will transmit both a data signal and a pilot signal to the mobile unit through the satellite. These signals are uplinked to the satellite at frequencies centered at $29.634 \mathrm{GHz}$, translated by the satellite to frequencies centered at $19.914 \mathrm{GHz}$, and retransmitted on the downlink to the mobile terminal. The mobile unit may be located at multiple sites in the United States, as will be discussed below. For the return link, the mobile unit transmits a data signal at frequencies centered at $29.634 \mathrm{GHz}$ up to the satellite. The satellite then translates this signal to frequencies centered at $19.914 \mathrm{GHz}$ and retransmits it to the fixed station.

\section{ACTS Overview}

ACTS, illustrated in Figure 2, is an experimental K/Ka-band satellite that has been developed by GE under contract to NASA. It is scheduled for launch in the summer of 1993 and will be placed in geostationary orbit at $100^{\circ}$ west longitude. In addition to its $\mathrm{K} / \mathrm{Ka}$-band operation, the ACTS has the following three hardware segments: (1) a multibeam antenna (MBA); (2) a baseband processor (BBP); and (3) a microwave switch matrix (MSM).

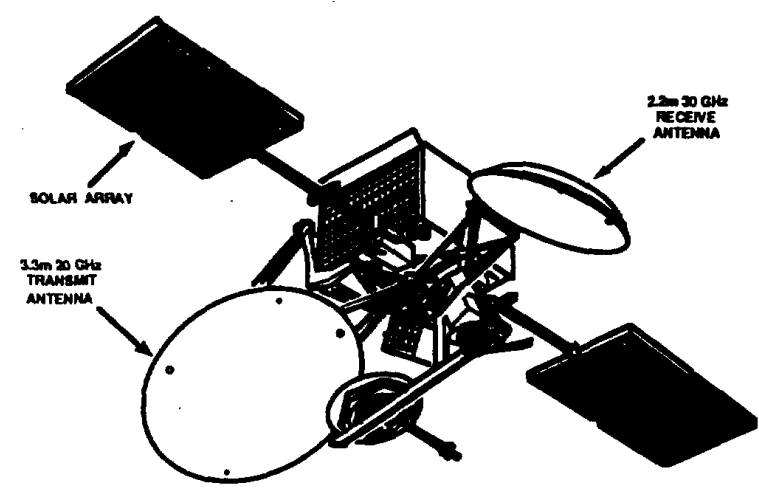

Figure 2 Advanced Communications Technology Satellite

The MBA consists of an uplink receive antenna and a downlink transmit antenna. Basic antenna designs are offset-fed Cassegrain configurations that have a subreflector in between the feed and the main reflector. The receive antenna uses a 3.3 meter main reflector to produce "spot" beams that are approximately 110 miles in diameter. The MBA produces 3 basic types of spot beams. First, there are 3 pairs of "fixed" spot beams to provide coverage for users in Cleveland, Atlanta, and Tampa. Second, there are two pairs of "hopping" beams that can be scanned "continuously" over two large sectors in the United States, as well as to discrete city locations throughout the United States (e.g., Houston, Los Angeles, etc.). This MBA coverage plan is shown in Figure 3. 


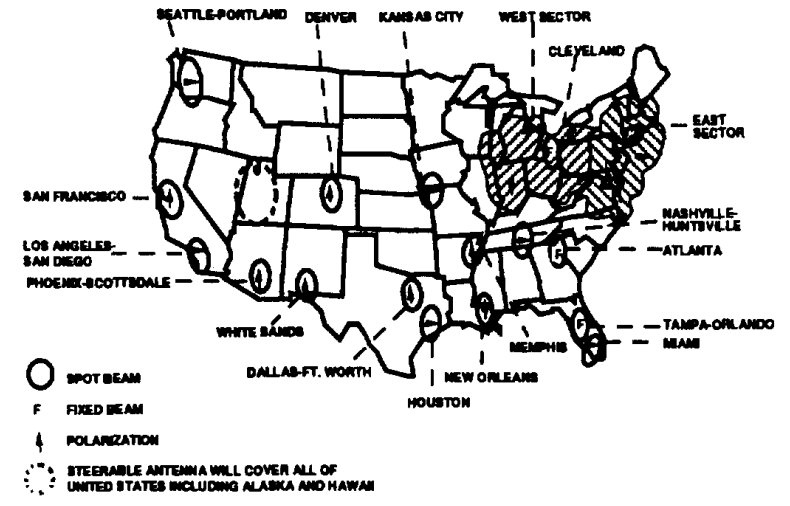

Figure 3 ACTS Spot Beam Coverage

Examination of this figure shows a somewhat larger spot beam produced by the ACTS' mechanically steerable antenna which uses a 1 meter reflector. As the name implies, this antenna can be "mechanically" steered to cover any position on the earth that is visible from the ACTS orbital position. The ACTS system allows terminals to communicate between any combination of beams.

During the BBP mode of operation, received signals are downconverted, demodulated to digital baseband, stored, decoded if necessary, routed to an appropriate output memory, encoded if needed, modulated, and passed on to the appropriate transmitting chain. The frequency upconverter and traveling-wave tube amplifier (TWTA) combinations translate the 3 GHz TDMA signals to $20 \mathrm{GHz}$, and amplify them to a power level suitable for downlinking to ground terminals. Transmission burst rates are 110 or $27.5 \mathrm{Mbps}$ on the uplink while all traffic is burst at $110 \mathrm{Mbps}$ on the downlink. The TDMA signals received and transmitted by the BBP are modulated using serial minimumshift keying (SMSK).

During the MSM mode of operation, the received signals are downconverted to an IF, routed to and switched by the MSM, and then passed to the appropriate upconverter and transmitter chain where the signals are upconverted to $20 \mathrm{GHz}$, amplified by the TWTA's, and transmitted. There is no onboard storage of the traffic, therefore the traffic must be routed and transmitted in real time. In this mode of operation, ACTS is operating as a bent pipe. The MSM mode of the satellite will be the mode utilized during the AAMT experiments and demonstrations.

\section{AMT Configurations}

Two land mobile variations of this terminal are under development. The first is the standard commercial configuration as shown in Figure 4. It consists of the basic AMT as developed by JPL. The second configuration is a military spin-off of the first. It is shown in Figure 5 . In addition to including the basic capabilities of the AMT, it has a SINCGARS radio interface as an alternate configuration. In this case, a SINCGARS radio is connected to the RF portion of the AMT. The mobile platform that both configurations of the terminal will be installed in is a HMMWV with a fully integrated Standardized Integrated Command Post Shelter (SICPS).

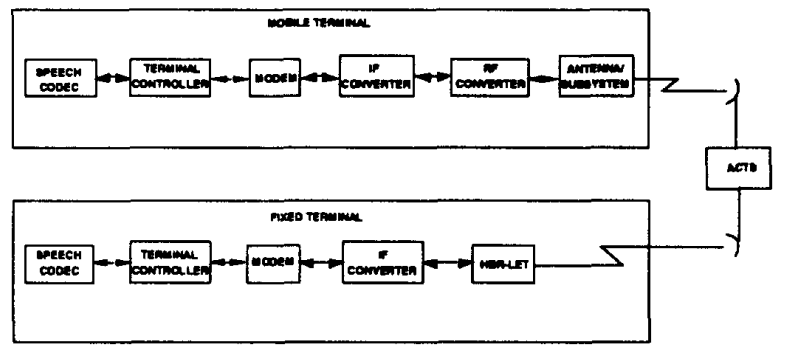

Figure 4 AMT and Fixed Terminal Block Diagrams

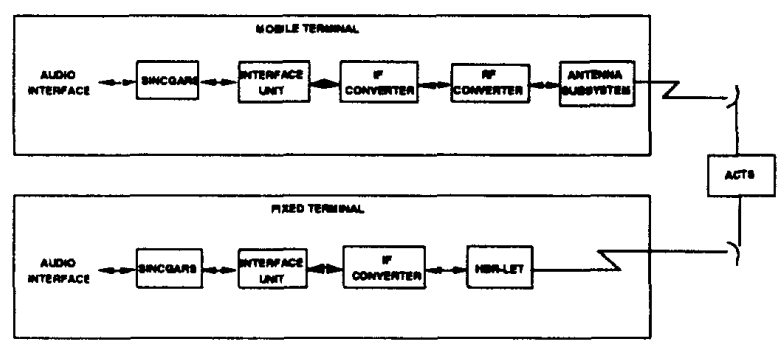

Figure 5 SINCGARS Based Mobile and Fixed Terminal Block Diagrams

\section{AMT Hardware}

The AMT consists of the following subsystems: a speech codec, a terminal controller, a modem, a transceiver consisting of an IF converter (IFC) and an RF converter (RFC), 
and the antenna subsystem. A description of these subsystems follows. A key feature of the AMT that is interwoven among several of the subsystems is the rain compensation algorithm (RCA) [4]. The basic premise of the RCA is that by lowering the data rate from 9.6 kbps to 4.8 or $2.4 \mathrm{kbps}$ in the advent of a rain event, the link margin can be increased by approximately $3 \mathrm{~dB}$ and $6 \mathrm{~dB}$, respectively. The RCA is a novel algorithm by which the AMT is able to dynamically adjust the data rate to help mitigate the effects of rain attenuation. The RCA utilizes pilot power measurements at the mobile terminal and satellite beacon power measurements at the fixed terminal to determine rain attenuation. The rain attenuation information is communicated to both terminals through the AMT communications protocol [5] and a conflict free decision as to whether the data rate should be lowered or raised is made.

The speech codec converts input analog speech signals to a compressed digital representation at data rates of 2.4, 4.8 and 9.6 kbps, with monotonically improving voice quality. The $2.4 \mathrm{kbps}$ compression algorithm is the government standard LPC-10, the $4.8 \mathrm{kbps}$ algorithm is the proposed CELP government standard, and at $9.6 \mathrm{kbps}$ an MRELP algorithm is adopted. Data rate switches are performed upon command from the terminal controller based on RCA information or upon user command. Data rate switching is performed with no user intervention and "on-the-fly" to have minimal impact on the continuity of the link. Independent transmit/receive data rates are supported by the codec. The fixed terminal codec is capable of interfacing to the Public Switched Telephone Network (PSTN), allowing the user to place a call to virtually any location with a telephone.

The terminal controller (TC) is the heart of the mobile terminal. It contains the algorithms that translate the communications protocol into the operational procedures and interfaces among the terminal subsystems. For example, it executes the timing and handshake procedures for the interaction among the speech codec, modem, user interface, and any external device (data source or sink) during link setup, relinquishment, or data rate change. The TC also contains the RCA routines and is responsible for executing them. The TC has control over the operation of the IFC and RFC and maintains high-level control over the antenna platform. The TC will support the test functions required during experimentation, such as bit stream generation, correlation and bit error counting.

The baseline AMT modem will implement a simple but robust DPSK scheme with rate $1 / 2$, constraint length 7 convolutional coding and interleaving. The performance of the modem at a data rate of $9.6 \mathrm{kbps}$ is a bit error rate (BER) of $10^{-3}$ at an $\mathrm{Eb}_{\mathrm{b}} / \mathrm{N}_{0}$ of $6.6 \mathrm{~dB}$ in AWGN with frequency offsets and including modem implementation losses. The modem has been designed to handle frequency offsets of $+/-10.0$ $\mathrm{kHz}$ without additional degradation. In addition to the 2.4, 4.8 and $9.6 \mathrm{kbps}$ rates the modem has been designed to handle up to $64 \mathrm{kbps}$ for the demonstration of high quality digital audio and slow scan compressed video. Essential to the modem design is a built-in robustness to deep, short-term shadowing. The modem will "free-wheel", i.e., not loose synchronization through a signal outage caused by road-side trees and will reacquire the data rapidly after such a drop-out.

The IFC translates between 3.373 and a lower $70 \mathrm{MHz}$ IF at the output/input of the modem. The IFC provides frequency selectivity on both the up and down conversion chains. The input/output bandwidth of the IFC at $3.373 \mathrm{GHz}$ is $300 \mathrm{MHz}$, with the capability to tune to 6000 different channels in this bandwidth with a $\mathbf{5 0}$ $\mathrm{kHz}$ resolution. A key function of the IF converter is pilot tracking and Doppler precompensation. The down-converted pilot is tracked in a phase-locked loop and used as a frequency reference in the mobile terminal. The tracked pilot is also processed in analog hardware and mixed with the up-converted data signal from the modem to pre-shift it to offset the Doppler on the return link. The IF converter provides the TC and antenna subsystem with pilot signal strength for RCA and antenna pointing operation respectively. The pilot in-phase and quadrature components are provided as outputs for link 
characterization.

The RFC simply translates the transmit signal from $3.373 \mathrm{GHz}$ to $29.634 \mathrm{GHz}$, and the receive signal from $19.914 \mathrm{GHz}$ to $3.373 \mathrm{GHz}$. The bandwidth of the RFC is $300 \mathrm{MHZ}$. THE RFC also provides the necessary power amplification for the transmit signal. The nominal output power of the RFC is 1.25 Watt, with a TWTA for experimental purposes providing up to 10 Watts.

The vehicle antenna is a critical Ka-band technology item in the AMT. Two types of antennas are being developed, though only the first will be demonstrated by the Army in the spring of 1994. The first is a "passive" elliptical reflector-type antenna [6] to be used in conjunction with a separate TWTA or a solid state power amplifier, and the second is an "active" array antenna with MMIC HPA's and LNA's integrated onto the array. Both antennas have their distinct advantages. The reflector is simpler and less risky and can accept up to $10 \mathrm{~W}$ of input power. The active array, despite being more complex and risky to develop, exploits MMIC technology to overcome some of the losses in the Ka-band hardware. The integration of the amplifiers also leads to a smaller more conformal antenna assembly. Both antenna will have a minimum EIRP of $22 \mathrm{dBW}, \mathrm{G} / \mathrm{T}$ of $-8 \mathrm{~dB} / \mathrm{K}$, and bandwidth of $300 \mathrm{MHz}$, over a 12 degree elevation beamwidth. Testing of the reflector antenna has found the actual minimum $G / T$ to be $-6 \mathrm{~dB} / \mathrm{K}$, over the elevation beamwidth. The antenna also achieves an EIRP of $32 \mathrm{dBW}$ over the elevation beamwidth, when the input power is $10 \mathrm{~W}$. The reflector will reside inside an ellipsoidal water-repelling radome of outside diameter 9" (at the base) and maximum height 3.5". The active array antenna will reside in slightly smaller radome. Both antenna are mechanically steered in the azimuthal plane.

The reflector antenna will be the baseline antenna utilized in the AAMT (the active array antenna will be completed in FY'95). This antenna is illustrated in Figure 6. The antenna will provide full 360 degree azimuthal coverage of the satellite in various spot beam locations in the continental United States. The $3 \mathrm{~dB}$ elevation beamwidth of approximately 19 degrees will provide sufficient elevation coverage to account for local road variations.

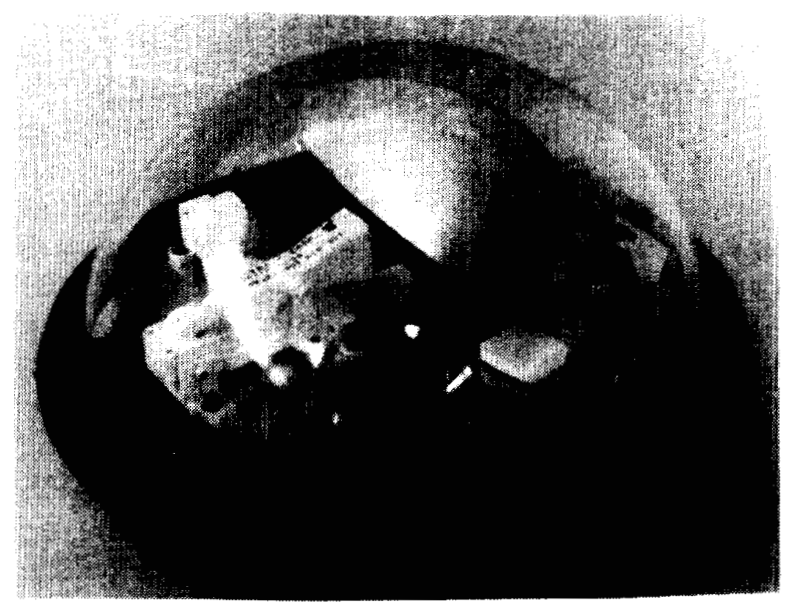

Figure 6 AMT Mechanically Steered Reflector Antenna

The antenna controller for the reflector antenna utilizes a mechanical dithering scheme where the antenna is smoothly dithered about its boresight by about a degree at a rate of $2 \mathrm{~Hz}$. The noncoherent pilot signal strength measured through this dithering process will be used to complement inertial information derived from a simple turn rate sensor. The combination of these two inputs is used to keep the antenna pointed at the satellite even if the satellite is shadowed for up to ten seconds. The primary information used for satellite acquisition is the received pilot signal strength. The primary information used for tracking the satellite is the inertial information from the rate sensor. This mechanical pointing scheme is one of the benefits of migration to Ka-band. The considerably smaller mass and higher gain achievable relative to $\mathrm{L}$-band make the mechanical dithering scheme feasible and obviate the need for additional RF components to support electronic pointing.

\section{AAMT SINCGARS Interface}

In order to permit SINCGARS operations with the AAMT, the equipment up to the modem 70 $\mathrm{MHz}$ input/output frequency will be replaced by the SINCGARS radio. The SINCGARS manpack/vehicular radio system is a VHF/FM 
fixed frequency and frequency hopping transceiver. The radio operates in the 30.0087.975 MHz band with $232025 \mathrm{kHz}$ channels. Both analog and digital voice are supported by the radio, and data rates to $16 \mathrm{kbps}$ are supported. The radio will be interfaced to the AMT IFC at a nominal $70 \mathrm{MHz}$ IF and the digital capability of the radio utilized. To ensure that the proper transmit and receive power levels are maintained, and to route the transmit and receive signals to the proper IFC/SINCGARS ports, an interface unit will be developed. The interface will consist of a simple RF switch that is keyed from the push to talk signal of the SINCGARS radio. Transmit signal attenuators will reduce the SINCGARS transmit levels to the appropriate IFC levels, as well as control the IFC receive levels to the appropriate SINCGARS levels. The normal mode of operation of the SINCGARS radio will be the fixed frequency mode, although the partial band frequency hop mode is also feasible.

The IFC will convert the $70 \mathrm{MHz}$ output of the SINCGARS to a $3.373 \mathrm{GHz}$ IF while transmitting and perform the reverse while receiving. The bandwidth of the IFC at $70 \mathrm{MHz}$ is $30 \mathrm{MHz}$ through which the SINCGARS could be operated in either a fixed frequency or hop mode. The RFC will translate the transmit signal from $3.373 \mathrm{GHz}$ to $29.634 \mathrm{GHz}$, and the receive signal from $19.914 \mathrm{GHz}$ to $3.373 \mathrm{GHz}$. The mechanically steered reflector antenna and associated antenna controller function as in the basic AMT design, providing the final RF stage of the terminal.

\section{Fixed Base Station Terminal}

Block diagrams of the fixed terminals for both the AMT configuration and the SINCGARS configuration are similar to those shown for the mobile units in Figures 4-5, respectively. The terminal either consists of the basic AMT equipment up through the IFC, or the SINCGARS and the IFC, plus the High Burst Rate-Link Evaluation Terminal (HBR-LET): In both terminal configurations, the equipment up through the IFC is identical to the mobile terminals except the pilot tracking capabilities of the IFC are not utilized. The HBR-LET provides the remainder of the RF chain, including a $4.7 \mathrm{~m}$ antenna. This terminal will be located at NASA LeRC in Cleveland, Ohio. The fixed terminal provides connectivity to the PSTN.

The HBR-LET has been designed to operated with the MSM mode of operation of ACTS. On the uplink portion of the HBR-LET, a two-stage upconverter converts a signal from $3.373 \mathrm{GHz}$ to $29.634 \mathrm{GHz}$. The uplink power is provided by a TWTA which has a saturated power output of 85 Watts. This TWTA is integrated with a $30 \mathrm{GHz}$ solid state amplifier and filtering to form the HBR-LET transmitter. The receiver portion of the HBR-LET consists of a four stage low noise amplifier at the front end. This is followed by a MMIC mixer and amplifier that converts the received signal from $19.914 \mathrm{GHz}$ to $3.373 \mathrm{GHz}$. In addition, the HBR-LET has been developed for rain compensation capabilities. The receive portion of the HBRLET tracks a beacon signal level. As this signal level varies due to atmospheric changes between ACTS and the fixed terminal, the transmitted signal power can be adjusted. The HBR-LET can compensate for atmospheric changes of up to approximately $20 \mathrm{~dB}$ (for the AAMT setup) without any noticeable degradation in the system performance.

\section{ARMY DEMONSTRATION PROGRAM}

The AAMT initiative will demonstrate and evaluate the reality of communications on the move using ACTS. Demonstrations will not be limited to basic voice communications, but may also include the transmission of weather, terrain, imagery, and intelligence data for use by the soldier. The overall system performance will be evaluated both quantitatively and qualitatively. The system performance will be characterized for various terrains at different $\mathrm{C} / \mathrm{N}_{0}$ 's (carrier power to noise power spectral density ratios) and frequency offsets.

The single most important thrust behind the AAMT program is to provide a mechanism for senior officials and requirements personnel to witness and experience comm-on-the-move first hand. To this end, demonstrations and 
actual hands-on use of the terminal will be coordinated with Army personnel at a number of military locations so as to gain maximum requirements exposure. The experiments and demonstrations will occur over a two month period of time at five different locations around the country. These demonstrations should begin in the spring of 1994 and be complete by summer. A complete listing of all of the planned demonstration locations, and the respective spot beams that will be utilized are provided in Table 1.

Table 1 Demonstration Locations and Spot Beam Requirements

\begin{tabular}{|l|c|}
\hline \multicolumn{1}{|c|}{ Location } & ACTS Spot Beam \\
\hline $\begin{array}{l}\text { Army Space Command, } \\
\text { Colorado Springs, Colorado }\end{array}$ & Denver \\
\hline Fort Leavenworth, Kansas & Kansas City \\
\hline Fort Monmouth, New Jersey & East Scan Sector \\
\hline Fort Monroe, Virginia & East Scan Sector \\
\hline Fort Gordon, Georgia & Steerable \\
\hline
\end{tabular}

\section{SUMMARY/CONCLUSIONS}

NASA's ACTS program provides the Army with an opportunity to investigate state-of-the-art satellite communications involving land-mobile terminals. The AAMT is a low risk ARMY/NASA/JPL collaboration to take NASA/JPL's already developed commercially oriented AMT and repackage it into a military HMMWV. The terminal will employ a very small mechanically steered reflector antenna. With it, the Army will be able to achieve previously unattainable mobile communications at 64 kbps. The Army eagerly awaits the demonstration and analysis of this terminal. Its performance has the potential to change Army communications doctrine. The antenna system's low cost and high efficiency may find a permanent place in the Army's antenna technology roadmap.

The AAMT program is only one part of a larger Army ACTS Demonstration Program intended to investigate new concepts and capabilities in satellite communications. The lessons learned from ACTS will apply to future commercial and military systems alike. Although ACTS contributes to the development of the commercial Ka frequency band as an asset in its own right, it also provides a technological stepping stone to the provision of similar services at even higher military frequencies; MILSTAR in particular.

\section{REFERENCES}

[1] F. Gargione, "The ACTS Spacecraft," Proceedings of the 14th AIAA International Communication Satellite Systems Conference, Washington, D.C., March 22-26, 1992, pp. 1146-1150.

[2] K. Dessouky, T. Jedrey, "The ACTS Mobile Terminal," Proceedings of the 14th AIAA International Communication Satellite Systems Conference, Washington, D.C., March 22-26, 1992, pp. 1758-1763.

[3] SINCGARS System Engineering Document - Ground Radio System, Prepared for the United States Army Communications Electronics Command, Fort Monmouth, New Jersey, Contract No. DAAB07-78-C-0150, by ITT Aerospace/Optical Division, July 28, 1989.

[4] E. H. Satorius, "Rain Compensation Algorithm for the ACTS Mobile Terminal System", JPL IOM no. AMT:331.5-92-094, Sept. 15, 1992.

[5] N. Lay and K Dessouky, "A Communication Protocol for Mobile Satellite Systems Affected by Rain Attenuation", IEEE J. Selected Areas in Commun., vol. 10, no. 6, August 1992.

[6] A. Densmore, V. Jamnejad, "A SatelliteTracking $\mathrm{K}$ and $\mathrm{Ka}$ Band Mobile Vehicle Antenna System," Submitted to the IEEE Transactions on Vehicular Technology. 